\title{
GMR
}

\section{Functional characterization and analysis of the Arabidopsis UGT71C5 promoter region}

\author{
Z. Liu' ${ }^{1,2,4}$, Q.H. Luo ${ }^{3}$, J.M. Wang ${ }^{1}$, X.F. Li' ${ }^{1}$ and Y. Yang ${ }^{1,2}$ \\ ${ }^{1}$ Key Laboratory of Bio-Resources and Eco-Environment of the Ministry of Education, \\ College of Life Sciences, Sichuan University, Chengdu, Sichuan, China \\ ${ }^{2}$ State Key Laboratory of Hydraulics and Mountain River Engineering, \\ Sichuan University, Chengdu, Sichuan, China \\ ${ }^{3}$ Longquanyi District Bureau of Agriculture Development, Chengdu, Sichuan, China \\ ${ }^{4}$ Department of Biotechnology, Chengdu Institute of Biological Products, Chengdu, \\ Sichuan, China \\ Corresponding author: Y. Yang \\ E-mail: yangyi19620528@126.com \\ Genet. Mol. Res. 14 (4): 19173-19183 (2015) \\ Received August 25, 2015 \\ Accepted October 31, 2015 \\ Published December 29, 2015 \\ DOI http://dx.doi.org/10.4238/2015.December.29.27
}

ABSTRACT. In the present study, we isolated an Arabidopsis promoter, UGT71C5, and analyzed its role in the regulation of the light response mechanism. We constructed a fusion vector pBI121-pU-GUS by integrating the UGT71C5 promoter upstream of the GUS reporter gene in $\mathrm{pBI} 121$, and then transferred this vector into Arabidopsis plants. The GUS activity of the transgenic plants was detected using a spectrophotometer under normal growth conditions as well as under light, drought, and ABA stress-treatments. The obtained results indicated that the GUS activity of transgenic plants ranged in between the activities observed in wild-type and 35S transgenic plants, which were used as positive control. Light stress for 8 and $12 \mathrm{~h}$ increased the GUS activity in transgenic plants by 3 and 4 times, respectively, compared to the activity in these plants under normal conditions. No such change in the GUS activity was observed under drought and ABA-treated conditions. This suggests that the UGT71C5 promoter is light inducible. Our study provides helpful insights into the elucidation of 
inducible promoters in Arabidopsis and the molecular mechanisms of light response.

Key words: Light stress; Promoter; GUS activity; Transgenic plant; Arabidopsis

\section{INTRODUCTION}

Light is one of the most important factors affecting plant growth and development. It is essential for morphogenesis, cellular and subcellular differentiation as well as gene expression in higher plants (Batschauer, 1998). It has been widely demonstrated that light-induced transcriptional regulation plays a vital role in plant growth and development (Tobin and Kehoe, 1994; Terzaghi and Cashmore, 1995; Millar and Kay, 1996). Thus, systematic identification and characterization of specific light-induced regulators and light-responsive elements (LREs) in plants aids in understanding the light-regulated mechanisms operative during plant growth and development.

Glycosyltransferases (EC 2.4.x.y) found in all organisms catalyze the transfer of sugar moieties from activated donor molecules to specific acceptor molecules (Campbell et al., 1997; Jones and Vogt, 2001). Many compounds or molecules can donate sugar moieties, such as di-or poly- saccharides, 1-phosphate sugars, nucleotide diphospho sugars (NDP sugars), and uridine diphosphate glucuronic acid. In plants, the most common donors of sugar moieties are UDP (uridine diphosphate)-galactose, UDP-rhamnose, UDP-xylose and UDP-glucuronic acid (Bar-Peled et al., 1991; Ishikura and Mato,1993; Martin et al., 1999; Miller et al., 1999; Jones et al., 2003; Sawada et al., 2005). Plant glycosyltransferases are generally called UDP-glycosyltransferases (UGT). Sugar acceptors in plants include proteins, lipids, all major classes of secondary metabolites, such as antibiotics, steroids, phenols, terpenes, cyanohydrins, plant hormones, alkaloids, plant toxins, and exogenous substances such as herbicides and pesticides, in addition to monosaccharides, oligosaccharides and polysaccharides (Vogt and Jones, 2000; Paquette et al., 2003; Lim and Bowles, 2004; Bowles et al., 2005; Gachon et al., 2005; Lim, 2005). UGTs catalyze the transfer of sugar moieties from activated donor molecules to specific acceptor molecules (Pflugmacher and Sandermann, 1998).

Promoter is one of the most important regulatory elements of gene expression, which determines the range and the level of expression as well as its tissue-, organ-, and developmental stage-specificity in higher plants. Currently, the most widely used promoters in plant genetic engineering are the constitutive promoters. These promoters, such as the CaMV $35 \mathrm{~S}$ promoter (Odell et al., 1985), the maize ubiquitin promoter, and the rice actin promoter, promote the expression of the exogenous genes in all the parts of transgenic plants during all the developmental stages. However, the constitutive expression of exogenous genes tends to cause abnormalities in plant morphology and affects their growth and development (Kohli et al., 1999). Inducible or tissue-specific promoters have attracted much attention and are thought to be more useful than constitutive promoters for effective expression of exogenous genes, without the adverse effects on the transgenic plants. A large number of specific or inducible-promoters that are seed-specific, fruit-specific, mesophyll cell-specific, root-specific, damage-inducible, chemical-induced, lightinduced, or heat shock-induced have been discovered in plants. The isolation and application of these promoters laid the foundation for specific and controlled expression of exogenous genes in host plants. 
It has been reported that over-expression of UGT71C5 may improve stress tolerance in Arabidopsis, although the molecular mechanism underlying this process is yet unclear. In the present study, we isolated the Arabidopsis UGT71C5 promoter and characterized its function in transgenic plants. Using Agrobacterium-mediated transformation, we transformed the fusion vector pBI121-pU-GUS into Arabidopsis plants and examined the GUS activities in the transgenic plants under different stress treatments. We observed that this promoter was light-inducible, which suggests that light can affect the stress-response of the plant, by affecting the expression of UGT71C5. The results obtained in this study could aid in understanding the role of inducible promoters in Arabidopsis, and also provides insights into the molecular mechanisms operating in the regulation of plant growth and development by environmental factors.

\section{MATERIAL AND METHODS}

\section{Plant materials and culture conditions}

The Arabidopsis ecotype RLD was used in this study. Arabidopsis plants were first grown on germination medium (GM) agar plates until the seedling stage and then transferred to culture pots containing nutrient soil and vermiculite (1:1 mixture) together with 1/2 MS medium (Murashige and Skoog, 1962). Plants (transgenic lines or RLD ecotype) were grown in a controlled chamber with long day light (16-h light/8-h dark) at $23^{\circ} \pm 2^{\circ} \mathrm{C}$, for 4 weeks.

\section{Isolation and cloning of UGT71C5 promoter}

Genomic DNA was isolated from the RLD ecotype of Arabidopsis thaliana using the CTAB (hexadecyltrimethylammonium bromide) method (Murray and Thompson, 1980). Based on the Arabidopsis genome sequences (available at www.arabidopsis.org), we designed the primer pair PU-F and PU-R (Table 1) to amplify the promoter regions of the UGT71C5 gene by PCR The amplified DNA fragment was then cloned into pMD18-T vector to generate pMD18-T-PU recombinants. After transforming the recombinants into E.coli strain $\mathrm{DH} 5 \alpha$, we selected the positive clones by PCR screening and sequenced the inserted fragment. The regulatory and cis-elements in the promoter sequence were analyzed using PlantCARE (Lescot et al., 2002) and PLACE (Higo et al., 1999) databases.

\begin{tabular}{|c|c|}
\hline Primer name & Sequence \\
\hline PU-F & 5'-АTСТААСАТTССТTTTCTAАACATA-3' \\
\hline PU-R & 5'-СТGTCTTCATCTCTTTACTT-3' \\
\hline GUS-F & 5'-AGAGATGCTCGACTGGGCAGATG-3' \\
\hline GUS-R & 5'-TACATTGACGCAGGTGATCGGAC-3' \\
\hline UGT71C5-F & 5'-GGAATTCCGTCCAAGAGAGTC-3' \\
\hline UGT71C5-R & 5'-TGACACGATCTCCAAAGTAACC-3' \\
\hline Actin-F & 5'-TGGGATGACATGGAGAAGAT-3' \\
\hline Actin-R & 5'-ATACCAATCATAGATGGCTGG-3' \\
\hline
\end{tabular}

\section{Plasmid construction and Arabidopsis transformation}

Both the plasmids, pBI121 and pMD18-T-PU, were digested with HindIII and Smal (SigmaAldrich, http://www.sigmaaldrich.com/). The pBI121 fragment without the CaMV 35S promoter and 
the UGT71C5 promoter fragment obtained from the digestion of pMD18-T-PU were recovered and ligated to form the fusion vector $\mathrm{pBI} 121-\mathrm{PU}-\mathrm{GUS}$. After checking the accuracy of the resultant vector by sequencing (Invitrogen, Beijing), we transferred it into competent Agrobacterium tumefaciens (strain EHA105) cells by a freeze-thaw method. The transformed Agrobacterium were selected on YEB-agar plates containing $100 \mathrm{mg} / \mathrm{L}$ kanamycin and $100 \mathrm{mg} / \mathrm{L}$ streptomycin. The recombinant Agrobacterium was then transformed into Arabidopsis RLD ecotype using the floral dip method (Clough et al., 1998). Simultaneously, the plasmid pBI121 containing the general CaMV 35S promoter was also transformed into Arabidopsis using the method described above to obtain the positive control plants.

TO generation seeds were collected and screened on MS medium plates supplemented with $50 \mathrm{mg} / \mathrm{L}$ kanamycin, after vernalization at $4^{\circ} \mathrm{C}$ for about 10 days. Selected transgenic lines were grown to T3 generation and used in the experiments. The seedlings of 4-week-old T3 transgenic Arabidopsis were sampled to isolate total RNA by RNAiso reagent (TaKaRa, Japan). The RNA was then reverse-transcribed to cDNA using PrimeScript RT-PCR Kit (TaKaRa). Using actin as the reference gene, the expression level of the GUS gene in PU transgenic, positive control, and wild-type Arabidopsis plants was tested using semi-quantitative PCR with GUS primers (Table 1). Total protein was extracted from all the lines and their GUS protein expression level was detected using the western blotting. The anti-GUS and the secondary antibody used for western blotting were purchased from commercial sources (Sigma, USA).

\section{Spectrophotometric estimation of GUS activity in transgenic plants}

Young leaves from 4-week-old transgenic Arabidopsis plants were sampled and ground into fine powder under liquid nitrogen. The powder was homogenized in $3 \mathrm{X}$ extraction buffer (50 mM sodium phosphate buffer, pH 7.0, 10 mM EDTA, 0.1\% Triton X-100, $10 \mathrm{mM} \beta$-mercaptoethanol, $1 \mathrm{mM}$ 4-nitrophenyl $\beta$-D-glucuronide (PNPG), $0.02 \% \mathrm{NaN}_{3}$, and $100 \mathrm{~g} / \mathrm{mL}$ BSA). The homogenate was centrifuged for $10 \mathrm{~min}$, at $4000 \mathrm{rpm}$ and supernatant was collected. The total protein concentration in the supernatant was determined using a spectrophotometer (Model 680, Bio-Rad, USA) by Coomassie brilliant blue method (Bradford, 1976). Supernatant containing $200 \mu$ total protein was aliquoted into six labeled tubes, and the extraction buffer was added to each tube to a final volume of $1 \mathrm{~mL}$. After proper mixing, the reaction in the first tube was terminated by adding the termination buffer (400 $\mu \mathrm{L} 1 \mathrm{M}$ 2-amino-2-methyl-propylene), which was followed by the addition of $400 \mu \mathrm{L}$ termination buffer to the second, third, fourth, fifth and sixth tubes at $5,10,15,30$, and $60 \mathrm{~min}$, respectively. Absorbance at $415 \mathrm{~nm}$ was measured for all the samples and GUS enzyme activity was calculated using the formula: GUS activity = amount of nitrophenol generated per minute $(\mathrm{nmol})$ / total protein amount $(0.2 \mathrm{mg})$.

\section{GUS activity in transgenic plants under stress conditions}

The transgenic Arabidopsis plants were grown for 4 weeks under normal conditions (light intensity $\sim 120 \mu \mathrm{mol} \cdot \mathrm{m}^{-2} \cdot \mathrm{s}^{-1}$, 16-h light/8-h dark, $22^{\circ} \mathrm{C}$, relative humidity $80 \%$ ) and then were administered stress treatments as follows: Light stress treatment; 8-h dark treatment followed by 8-h and 12-h light treatment, respectively. Plants without light treatment was the control group; Drought treatment: plants were exposed to drought for 7 days; ABA treatment: plants were grown in MS liquid medium containing $10 \mu \mathrm{M}$ ABA for $10 \mathrm{~h}$. Samples from all the treated plants were collected to detect the GUS activity. 


\section{Identification of UGT71C5 expression in stressed conditions}

In order to detect UGT71C5 gene expression, we isolated total RNA from the leaves of 4-week-old Arabidopsis plants subjected to dark, light, drought, and ABA treatment for 10 days. The cDNA was synthesized using the PrimeScript ${ }^{\circledR}$ RT reagent Kit with gDNA Eraser kit (Takara, Japan), and qRT-PCR was performed using the SYBR ${ }^{\circledR}$ Premix ExTaq ${ }^{\mathrm{TM}}$ II kit (TaKaRa) on a BioRad IQ5 System (Bio-Rad). The primers used for qRT-PCR were designed on the basis of the Arabidopsis UGT71C5 gene sequence. The Arabidopsis actin gene was used as the reference (Table 1).

\section{RESULTS}

\section{Isolation and analysis of the Arabidopsis UGT71C5 promoter}

Based on the Arabidopsis genomic information (www.arabidopsis.org), UGT71C5 is located on chromosome $1(2,223,691-2,225,452)$. We designed a pair of primers to amplify a 1020-bp fragment upstream of the translation start site of UGT71C5 by PCR. The cis-elements in the promoter sequence were analyzed using the PlantCARE and PLACE databases, and the transcription start site was predicated (Figure 1). A putative TATA box and its corresponding potential transcription start site were found in the promoter sequence. The TATA box was located $120 \mathrm{bp}$ upstream of the transcription start site. The CAAT box, which is a common cis-acting element in promoter and enhancer regions, was found at -241 bp. In addition, eight GT1 [G(A/G) $(A / T) A A(A / T)$ ], two DRE (ACGT), MYB element and MYC elements were also identified (Table 2).

\begin{tabular}{lcc}
\multicolumn{2}{c}{ Table 2. Cis-acting elements in promoter of UGT71C5. } & Sequence \\
\hline Name & Distributing site & TATAA \\
\hline TATA BOX & -120 & CAAT \\
CAAT BOX & -241 & G(A/G)(A/T)AA(A/T) \\
GT1 & $-653,-654,-596,-574,-552,-530,+169,+182$ & ACGT \\
ERD1 & $-671,-426$ & (A/T)AACCA \\
MYB1 & -269 & CACATG \\
MYC & +99 & GAGAC \\
SURECORE & -321 & TTGAC \\
W BOX & $-667,-333$ & \\
\hline
\end{tabular}

\section{Identification of the function of UGT71C5 promoter in transgenic plants}

To investigate the function of the UGT71C5 promoter, we constructed the fusion vector pBI121-PU-GUS and transformed it into the Arabidopsis wild type (WT ecotype). All harvested transgenic seeds were screened on MS plates containing $50 \mathrm{mg} / \mathrm{L}$ kanamycin, to eliminate nontransgenic plants. Three elite transgenic lines (PU-1, PU-2, and PU-3) were obtained after the transformation. We analyzed GUS expression in the transgenic lines by semi-quantitative PCR using primers specific for GUS. Strong GUS expression was observed for seedlings that were transgenic for $35 \mathrm{~S}$ promoters, while no expression was observed in the wild-type seedlings. The seedlings that were transgenic for the UGT71C5 promoter showed GUS expression that was intermediate in intensity between the wild-type and 35S promoter transgenic lines (Figure 2). We also used western blot analysis to detect the GUS protein expression. Results indicated that the 
35S transgenic lines had the highest GUS protein expression, with medium expression in the lines of UGT71C5 transgenics, and no expression in the wild-type plants (Figure 3).

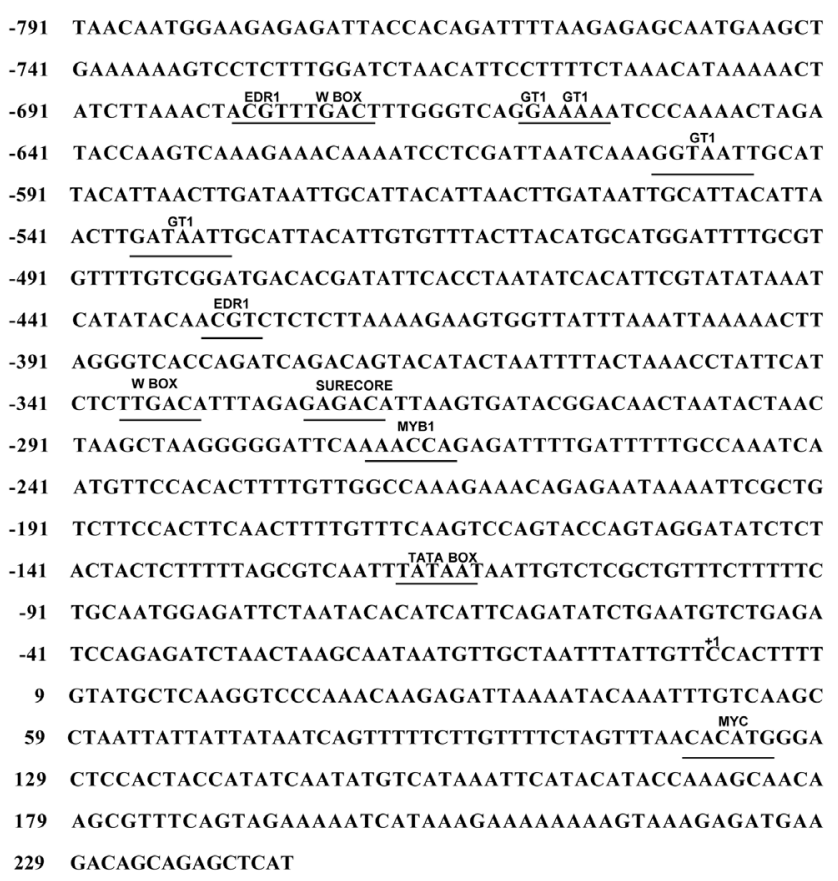

Figure 1. Sequence analysis of the UGT71C5 promoter.

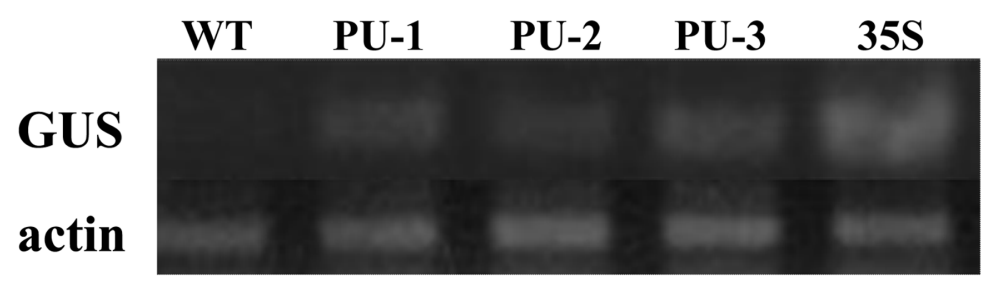

Figure 2. Expression level of the GUS gene in different transgenic lines detected by semi-quantitative PCR. WT: wildtype plants; PU1-PU3: UGT71C5 promoter transgenic lines; 35S: 35S transgenic plants.

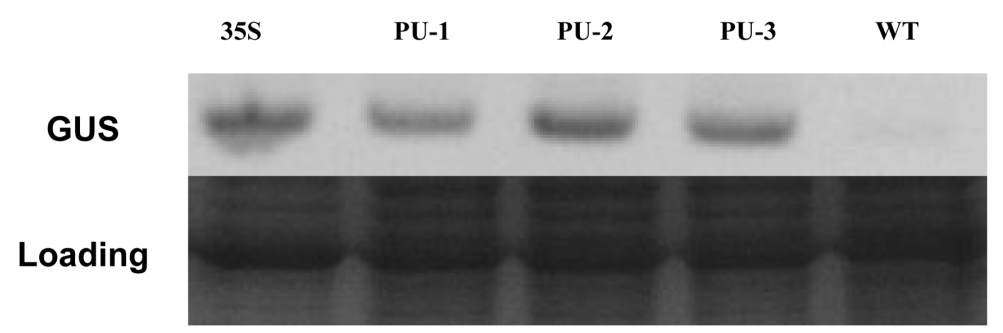

Figure 3. Detection of GUS expression in transgenic plants by western blotting. WT: wild-type plants; PU-1-PU-3: UGT71C5 promoter transgenic lines; $35 \mathrm{~S}$ : $35 \mathrm{~S}$ transgenic plants. 
To confirm these results, we further detected GUS activity in the transgenic plants by measuring the amount of nitrophenol generated, which is an indication of GUS activity, as described in the material and methods section. Three transgenic lines, PU-1, PU-2 and PU-3 displayed 1.93, 1.85 , and $2.01 \mathrm{nmol} \cdot \mathrm{min}^{-1} \cdot \mathrm{mg}^{-1} \mathrm{GUS}$ activity, respectively, with an average of $1.93 \mathrm{nmol} \cdot \mathrm{min}^{-1} \cdot \mathrm{mg}^{-1}$, which was higher than the activity observed in the wild-type line $\left(0.51 \mathrm{nmol} \cdot \mathrm{min}^{-1} \cdot \mathrm{mg}^{-1}\right)$, but much lower than that in the $35 \mathrm{~S}$ promoter lines $\left(11.53 \mathrm{nmol} \cdot \mathrm{min}^{-1} \cdot \mathrm{mg}^{-1}\right)$. These results were consistent with the semi-quantitative PCR analysis, which indicated that the UGT71C5 promoter could drive the expression of GUS in transgenic plants but to a level much lower than in the positive control plants, implying that the UGT71C5 promoter had a relatively lower transcriptional efficiency than the CaMV 35S promoter and thus is a weak promoter.

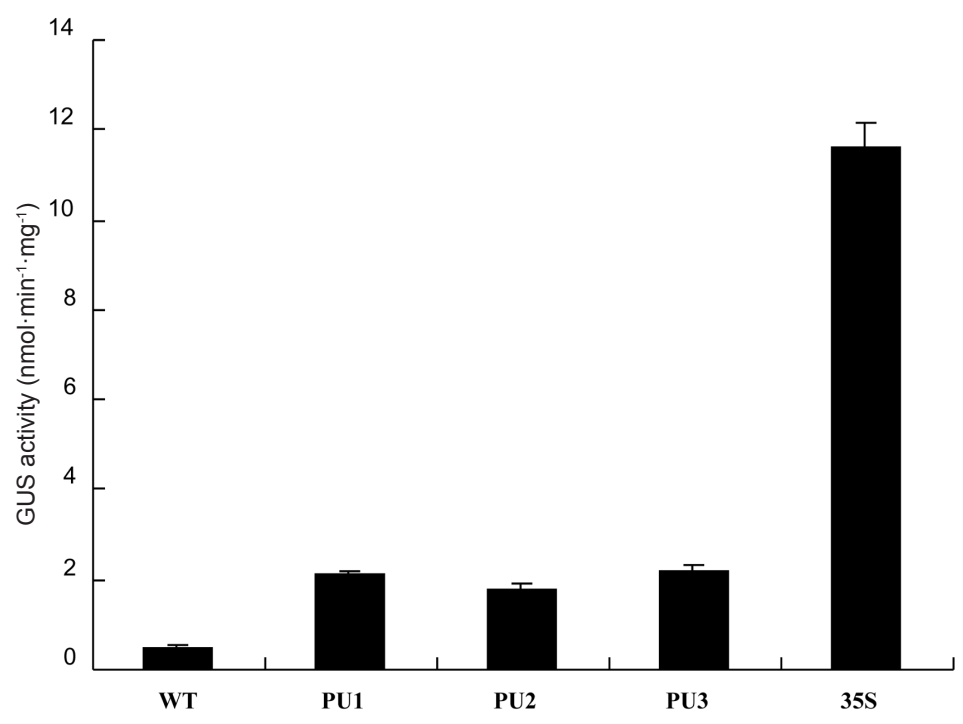

Figure 4. GUS activity in the transgenic plants measured by spectrophotometer. WT: wild-type plants; PU1-PU3: the UGT71C5 promoter transgenic lines; 35S: the 35S transgenic plants.

\section{Analysis of GUS activity in the transgenic lines under light-stressed conditions}

Our results indicated that the light responsive element GT1 was present in the promoter region of UGT71C5. We investigated GUS activity in the transgenic lines under light-stressed conditions (Figure 5). Under normal conditions (0 h), transgenic lines PU-1, PU-2, and PU-3 showed 2.01, 1.98, and $2.05 \mathrm{nmol} \cdot \mathrm{min}^{-1} \cdot \mathrm{mg}^{-1}$ GUS activity, respectively, while the wild type and positive control (35S) showed 0.51 and $11.53 \mathrm{nmol} \cdot \mathrm{min}^{-1} \cdot \mathrm{mg}^{-1}$ activity, respectively. When $8 \mathrm{~h}$ of strong light treatment was administered, the GUS activity increased to $5.12,5.03$, and $5.15 \mathrm{nmol} \cdot \mathrm{min}^{-1} \cdot \mathrm{mg}^{-1}$, respectively, which was 3 times higher than that under the normal conditions. The GUS activity of PU-1, PU-2 and PU-3 under 12-h light treatment increased to $6.09,5.94$ and $6.01 \mathrm{nmol} \cdot \mathrm{min}^{-1} \cdot \mathrm{mg}^{-1}$, respectively. However, the GUS activity in the wild type and the $35 \mathrm{~S}$ transgenic lines did not significantly differ between the normal and the light treated conditions. These results suggest that the UGT71C5 promoter is an inducible promoter that is set in motion by light. 


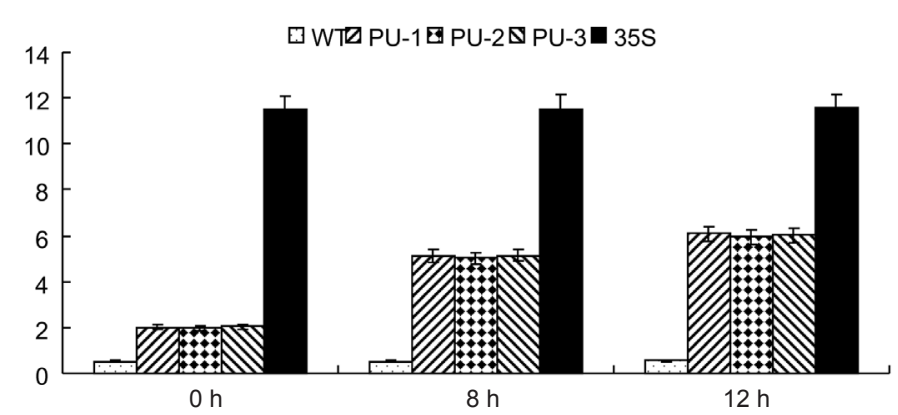

Figure 5. GUS activity analysis of the transgenic plants under light stress. WT: wild-type plants; PU1-PU3: UGT71C5 promoter transgenic lines; 35S: $35 \mathrm{~S}$ transgenic plants.

\section{Analysis of GUS activity in the transgenic lines under drought and ABA treatment}

The drought-associated motif ERD and the ABA-associated motif MYB/MYC were also found in the UGT71C5 promoter. Therefore, we further evaluated the GUS activity in the transgenic lines under drought conditions and ABA treatment (Figure 6). GUS activity was 1.98, 2.03, and $2.01 \mathrm{nmol} \cdot \mathrm{min}^{-1} \cdot \mathrm{mg}^{-1}$ for the PU-1, PU-2 and PU-3 lines, respectively, before the drought treatment, while it changed to $2.03,2.05$, and $2.01 \mathrm{nmol} \cdot \mathrm{min}^{-1} \cdot \mathrm{mg}^{-1}$ under drought stress conditions. Similarly, the values of the wild type under normal and drought conditions was 0.56 and $0.57 \mathrm{nmol} \cdot \mathrm{min}^{-1} \cdot \mathrm{mg}^{-1}$, respectively, while that for $35 \mathrm{~S}$ transgenic lines was 11.98 and $11.96 \mathrm{nmol} \cdot \mathrm{min}^{-1} \cdot \mathrm{mg}^{-1}$, respectively. This demonstrated that there was no significant change in GUS activity in the wild-type and positive control transgenic plants under normal and drought stress conditions. Under ABA treatment, the GUS activity of PU-1, PU-2 and PU-3 was $2.02,2.03$ and $2.15 \mathrm{nmol} \cdot \mathrm{min}^{-1} \cdot \mathrm{mg}^{-1}$, respectively, that of wild type was 0.56 , and that of $35 \mathrm{~S}$ lines was $12.07 \mathrm{nmol} \cdot \mathrm{min}^{-1} \cdot \mathrm{mg}^{-1}$. No significant change was observed under ABA treatment as well, suggesting that the UGT71C5 promoter was not induced by drought and ABA.

A

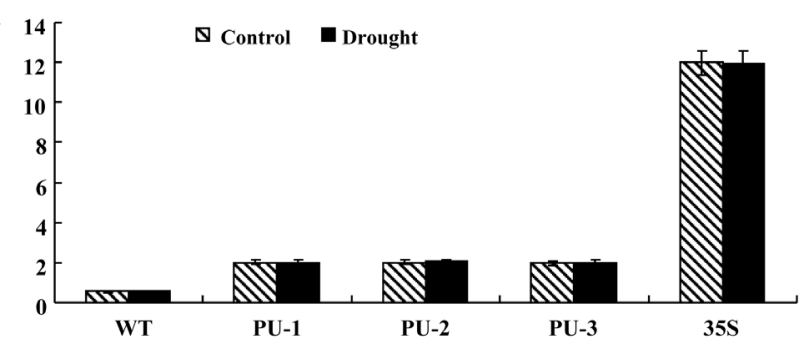

B

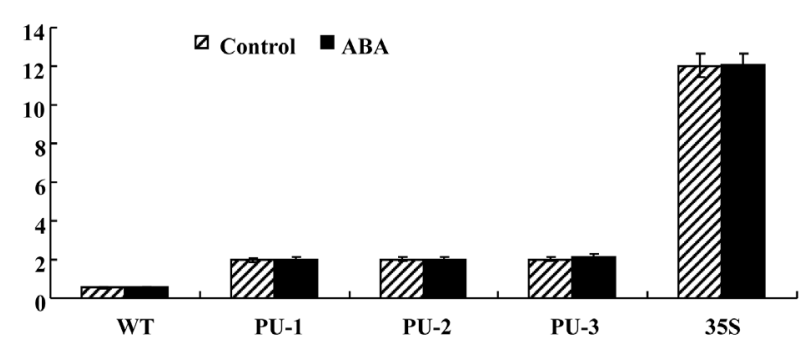

Figure 6. GUS activity analysis of the transgenic plants under drought (A) and ABA treatment (B). WT: wild-type plants; PU1-PU3: UGT71C5 promoter transgenic lines; 35S: 35S transgenic plants. 


\section{Light induced increases in the expression of UGT71C5}

According to the above analyses, UGT71C5 promoter was induced by light, but not by drought and ABA. To verify this result, we further evaluated the expression of UGT71C5 gene using qRT-PCR under different stress conditions (Figure 7). Results indicate that the expression of UGT71C5 increased by $80 \%$ under light treatment, while it decreased significantly under dark conditions. No significant changes were, however observed, under drought and ABA treatment, which was consistent with the GUS activity analysis. These results further demonstrated that the UGT71C5 promoter was light-inducible.

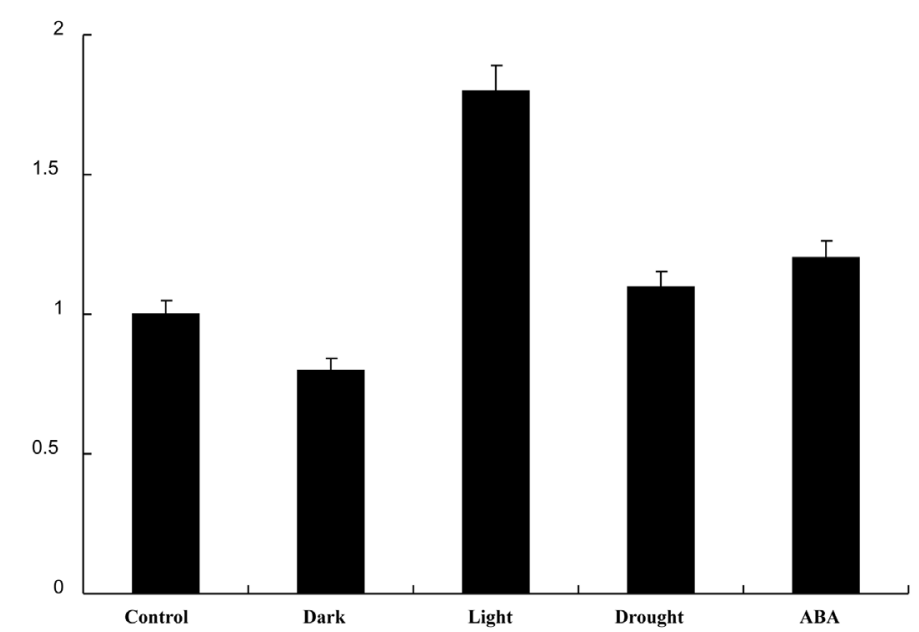

Figure 7. Expression level of UGT71C5 gene under various stress conditions as assessed by qRT-PCR analysis. WT: wild-type plant; PU1-PU3: the UGT71C5 promoter transgenic lines; 35S: the 35S transgenic plants.

\section{DISCUSSION}

In the present study, we isolated and analyzed the promoter of UGT71C5 gene in $A$. thaliana. Sequence analysis revealed several stress-associated cis-elements in the promoter. It has been demonstrated that GT1 is a light response-associated element, also known as II Box. It has GGTTAA motif, the common binding site for many light-regulated genes which could be bound by the TFIIA-TBP-TATA element to form a more stable complex (Zhou, 1999). A number of light-inducible promoters have a cluster of GT1 boxes, which, through an appropriate spatial distribution, makes them light responsive. During the light adjustment process, the GT1 box, GATA elements, and their flanking sequences work together as regulators of gene expression. Depending on the position and their combination with other elements, the GT1 box could act as a positive or negative regulatory element with its regulatory strength correlated with the number of GT1 boxes. The tobacco rbcMT-T promoter contains 7 GT1 motifs. When all the 7 motifs are present, this promoter drives the GUS gene expression in the dark, while when only 2 GT1 motifs are present, it drives the expression in the light (Mazarei et al., 1998).

In addition, other stress-associated regulatory elements have also been reported. The ERD motif plays a vital role in drought response in A. thaliana (Simpson et al., 2003). MYB and MYC 
are the drought and ABA-associated cis-elements (Abe et al., 1997, 2003; Busk et al., 1998). MYB transcription factor is widely present in plants, which combines with MYB genes in the regulatory process of plants' response to environment stress. In Arabidopsis, all stress-related genes contain the MYB element, such as rd22, rd17, and rd29. MYC elements are a kind of cis-acting elements involved in drought and ABA responses. MYB and MYC transcription factors combine together to regulate the expression level of $A B A$ response-associated genes.

In the present study, we constructed the fusion vector of UGT71C5 promoter and the GUS gene and then transformed it into Arabidopsis. Using semi-quantitative PCR and GUS activity analysis, we observed that the UGT71C5 promoter can regulate the GUS gene expression and further demonstrated that it was a light-inducible promoter. In comparison to $35 \mathrm{~S}$ transgenic plants, the UGT71C5 transgenic plants showed much weaker GUS activity. We postulate that the difference in the position of TATA box (generally located $32 \pm 7$ upstream of the transcription start site) in the UGT71C5 promoter compared to other eukaryotic promoters could account for the weaker activity, as the difference in the position affects the binding affinity of TFIID complex with the TATA box thereby resulting in a decreases activity of the promoter (Hahn et al., 1989). Furthermore, although light increased the expression of UGT71C5, the increase was limited, which suggests that UGT71C5 is a weak promoter.

Light is an important environmental factor for plant growth and development, which not only provides the energy for photosynthesis, but also regulates plant morphogenesis, an important developmental signal in almost all stages of the life cycle, from seed germination to flowering. The glycosyltransferase gene family is widely distributed in plants. They can modify the secondary metabolites of plant cells by glycosylation, contributing to the natural synthesis, modification, transport, and metabolism of a variety of natural products in plants. It has been found that overexpression of Arabidopsis UGT71C5 can improve glucose tolerance, which is correlated with the stress response (Yan et al., 2010). Additionally, other members of the UGT71C5 family are widely involved in a variety of physiological activities in plants, for example; UGT71B6 is involved in the ABA metabolism. Therefore, it is postulated that light as an environmental factor is involved in the stress response in Arabidopsis. This study fills an important gap in the knowledge about the impact of light on stress response in plants and paves the way for further scrutiny of the molecular mechanisms involved in the process.

\section{ACKNOWLEDGMENTS}

Research suported by the National Natural Science Foundation of China (Grant \#31171586 to Y. Yang and \#31271758 to J.M. Wang) and the National "973" project (\#2013CB733903).

\section{REFERENCES}

Abe H, Yamaguchi-Shinozaki K, Urao T, Iwasaki T, et al. (1997). Role of Arabidopsis MYC and MYB homologs in drought-and abscisic acid-regulated gene expression. Plant Cell 15: 1859-1868.

Bar-Peled M, Lewinsohn E, Fluhr R and Gressel J (1991). UDP-rhamnose: flavanone-7-O-glucoside-2"-O-rhamnosyltransferase. Purification and characterization of an enzyme catalyzing the production of bitter compounds in citrus. J. Biol. Chem. 266: 20953-20959.

Batschauer A (1998). Photoreceptors of higher plants. Planta 206: 479-492.

Bowles D Isayenkova J, Lim EK and Poppenberger B (2005). Glycosyltransferases: managers of small molecules. Curr. Opin. Plant Biol. 8: 254-263.

Bradford MM (1976). A rapid and sensitive method for the quantitation of microgram quantities of protein utilizing the principle 
of protein-dye binding. Anal. Biochem. 7: 248-254.

Busk PK and Pages M (1998). Regulation of abscisic acid-induced transcription. Plant Mol. Biol. 37: 425-435.

Campbell J, Davies GJ, Bulone V and Henrissat B (1997). A classification of nucleotide-diphospho-sugar glycosyltransferases based on amino acid sequence similarities. Biochem. J. 326: 929-939.

Clough SJ and Bent AF (1998). Floral dip: a simplified method for Agrobacterium-mediated transformation of Arabidopsis thaliana. Plant J. 16: 735-743.

Hahn S, Buratowski S, Sharp PA and Guarente L (1989). Yeast TATA-binding protein TFIID binds to TATA elements with both consensus and nonconsensus DNA sequences. Proc. Natl. Acad. Sci. U. S. A. 86: 5718-5722.

Higo K, Ugawa Y, Iwamoto M and Korenaga T (1999). Plant cis-acting regulatory DNA elements (PLACE) database: 1999. Nucleic Acids Res. 27: 297-300.

Ishikura N and Mato M (1993). Partial purification and some properties of flavonol 3-O-glycosyltransferases from seedlings of Vigna mungo, with special reference to the formation of kaempferol 3-O-galactoside and 3-O-glucoside. Plant Cell Physiol. 34: 329-335.

Jones P and Vogt T (2001). Glycosyltransferases in secondary plant metabolism: tranquilizers and stimulant controllers. Planta 213: $164-174$.

Jones P, Messner B, Nakajima J, Schäffner AR, et al. (2003). UGT73C6 and UGT78D1, glycosyltransferases involved in flavonol glycoside biosynthesis in Arabidopsis thaliana. J. Biol. Chem. 278: 43910-43918.

Lescot M, Déhais P, Thijs G, Marchal K, et al. (2002). PlantCARE, a database of plant cis-acting regulatory elements and a portal to tools for in silico analysis of promoter sequences. Nucleic Acids Res. 30: 325-327.

Lim EK (2005). Plant glycosyltransferases: their potential as novel biocatalysts. Chemistry 11: 5486-5494.

Lim EK and Bowles DJ (2004). A class of plant glycosyltransferases involved in cellular homeostasis. EMBO J. 23: 2915-2922.

Lim EK, Baldauf S, Li Y, Elias L, et al. (2003). Evolution of substrate recognition across a multigene family of glycosyl transferases in Arabidopsis. Glycobiology 13: 139-145.

Kohli A, Griffiths S, Palacios N, Twyman RM, et al. (1999). Molecular characterization of transforming plasmid rearrangements in transgenic rice reveals a recombination hotspot in the CaMV $35 \mathrm{~S}$ promoter and confirms the predominance of microhomology mediated recombination. Plant J. 17: 591-601.

Martin RC, Mok MC and Mok DW (1999). A gene encoding the cytokinin enzyme zeatinO-xylosyltransferase of Phaseolus vulgaris. Plant Physiol. 120: 553-558.

Mazarei M, Ying Z and Houtz RL (1998). Functional analysis of the Rubisco large subunit ÂN-methyltransferase promoter from tobacco and its regulation by light in soybean hairy roots. Plant Cell Rep. 17: 907-912.

Millar AJ and Kay SA (1996). Integration of circadian and phototransduction pathways in the network controlling CAB gene transcription in Arabidopsis. Proc. Natl. Acad. Sci. U. S. A. 93: 15491-15496.

Miller KD, Guyon V, Evans NS, Shuttleworth WA, et al. (1999). Purification, cloning, and heterologous expression of a catalytically efficient flavonol 3-O-galactosyltransferase exp ressed in the male gametophyte of Petunia hybrida. J. Biol. Chem. 274: 34011-34019.

Odell JT, Ferenc $\mathrm{N}$ and Chua NH (1985). Identification of DNA sequences required for activity of the cauliflower mosaic virus 35 S promoter. Nature 313: 810-812.

Paquette S, Moller BL and Bak S (2003). On the origin of family 1 plant glycosyltransferases. Phytochemistry. 62: 399-413.

Regev-Shoshani G, Shoseyov O, Bilkis I and Kerem Z (2003). Glycosylation of resveratrol protects it from enzymic oxidation. Biochem. J. 374: 157-163.

Sawada S, Suzuki H, Ichimaida F, Yamaguchi MA, et al. (2005). UDP-glucuronic acid: anthocyanin glucuronosyltransferase from red daisy (Bellis perennis) flowers. Enzymology and phylogenetics of a novel glucuronosyltransferase involved in flower pigment biosynthesis. J. Biol. Chem. 280: 899-906.

Simpson SD, Nakashima K, Narusaka Y, Seki M, et al. (2003). Two different novel cis-acting elements of erd1, a clpA homologous Arabidopsis gene function in induction by dehydration stress and dark-induced senescence. Plant J. 33: 259-270.

Tobin EM and Kehoe DM (1994). Phytochrome regulated gene expression. Sem. Cell Biol. 5: 335-346.

Yan JP, He H, Zhang JJ, Liu Z, et al. (2010). Overexpression of UDP-glucosyltransferase 71C5 increases glucose tolerance in Arabidopsis thaliana. Russian J. Plant Phys. 57: 875-881.

Zhou DX (1999). Regulatory mechanism of plant gene transcription by GT-elements and GT-factors. Trends Plant Sci. 4: 210-214. 